\title{
Diversidad de la vegetación y características morfoedáficas de las riberas de arroyos pampeanos
}

\author{
Patricia Gantes ${ }^{1, \otimes}$; Liliana Falco ${ }^{2}$ \& Aníbal Sánchez Caro ${ }^{1}$ \\ ${ }^{1}$ Grupo de Investigación en Ecología Acuática, Departamento de Ciencias Básicas, Universidad Nacional de Luján. Instituto \\ de Ecología y Desarrollo Sustentable (INEDES) UNLu-CONICET. ${ }^{2}$ Programa de Investigación en Ecología Terrestre, \\ Departamentode Ciencias Básicas, Universidad Nacionalde Luján. InstitutodeEcología y DesarrolloSustentable(INEDES)
} UNLu-CONICET.

\begin{abstract}
RESUMEN. Las riberas son ecosistemas espacial y temporalmente heterogéneos que favorecen una alta diversidad, pero que se podrían ver afectadas por la intensificación de ciertas perturbaciones antrópicas. Con el propósito de aportar información de base, que permita evaluar los efectos sobre la biodiversidad de eventuales disturbios, se caracteriza la vegetación por sus grupos biológicos y se analizan tres aspectos: la diversidad a tres escalas (margen, tramo y cuenca), su relación con características topográficas y edáficas y los cambios asociados a la presencia de ganado. Se identificaron 130 taxa; entre ellas, Cynodon dactylon y Eleocharis spp. tuvieron una frecuencia cercana al $100 \%$ y fueron los de mayor cobertura. Se halló $51 \%$ de especies herbáceas perennes, $27 \%$ de hidrófilas, $18 \%$ de anuales y $3 \%$ de árboles. En cuanto al origen, la proporción de exóticas no es menor a la mencionada para pastizales pampeanos, aunque las hidrófilas, propias de estos ambientes, son casi en su totalidad nativas. La diversidad beta calculada como el cociente entre tramo y márgenes fue 1.37, y entre cuenca y tramos fue 1.89. Por esto, la diversidad beta contribuye de manera importante a la biodiversidad regional. La diversidad se correlacionó con el eje que representa las variables edáficas en un análisis de componentes principales, a pesar que las pendientes explicaron una mayor proporción de la variabilidad entre sitios. La vertiente del Río de la Plata es la más diversa, y en un análisis discriminante se separó del resto. La densidad aparente del suelo fue la variable que más contribuyó a la distinción. Se sugiere que al momento de diseñar investigaciones y estrategias de manejo, la interpretación del funcionamiento de las riberas se realice en la escala de cuenca.
\end{abstract}

[Palabras clave: riqueza específica; grupos biológicos; escalas espaciales]

\begin{abstract}
Aвstract. Riparian vegetation diversity and morphoedaphic attributes in pampean streams. Riparian areas are temporally and spatially heterogeneous ecosystems which favor high species' diversity; however, they may be affected by the intensification of certain anthropogenic perturbations. With the aim of generating basic information to assess the effects of possible disturbances, riparian vegetation is characterized in terms of growth form, and three of its aspects are analyzed: diversity at three scales (bank, reach, and watershed), relationship with topographic and soil variables, and changes associated with livestock presence. In this study, 130 taxa were identified; among them, Cynodon dactylon and Eleocharis spp. exhibited frequencies close to $100 \%$ and the highest coverage level. Of all the species, $51 \%$ were perennials, $27 \%$ macrophytes and amphibious plants, $18 \%$ annual plants and 3\% trees. The proportion of exotic species is not lower than that of pampean grasslands, but macrophytes and amphibious plants are almost all natives. Beta diversity, calculated as the ratio between reach and stream-banks, was 1.37, and for watershed/reachs, 1.89 ; thus, beta diversity contributes significantly to regional biodiversity. In a principal component analysis, bank slopes explained the greater proportion of variability among sites. Nevertheless, diversity was correlated to the soil-variables axis. The Río de la Plata watershed was the most diverse, and was differentiated from the other watersheds in a discriminant analysis, primarily on the basis of soil-bulk density. We suggest that the functioning of riparian areas be considered at the watershed scale for the design of research projects and management strategies.
\end{abstract}

[Keywords: species richness; biological groups; spatial scales]

\section{INTRODUCCIÓN}

Las riberas de ríos y arroyos son ambientes altamente heterogéneos, ligados por un lado a una dinámica fluvial que determina gradientes transversales, longitudinales y verticales, así como también a procesos y disturbios en las terrazas adyacentes (Naiman et al. 1993; Poff et al. 1997; Ward et al. 2002; Gurnell 2014). Funcionalmente, la vegetación de las riberas interviene en la estabilización de las márgenes,

Editora asociada: Irina Izaguirre

gantespat@yahoo.com.ar en la protección frente a crecientes, en la retención de sedimentos y en el control de los flujos de agua, materia orgánica, nutrientes y contaminantes hacia los arroyos (Osborne and Kovacic 1993; Hood and Naiman 2000; Giling et al. 2014). La heterogeneidad espacial y temporal crea una gran variedad de hábitats para especies con diferentes requerimientos, lo que favorece una alta diversidad, a menudo mayor que la de las áreas adyacentes más elevadas (Naiman et al. 2000; Nilsson and

Recibido: 25 de abril de 2016

Aceptado: 26 de diciembre de 2016 
Svedmark 2002; Young-Mathwes et al. 2010). Esa diversidad es en parte producto de que las riberas funcionan como corredores de flora y fauna en el paisaje de las cuencas. De este modo, es importante discernir si la diversidad regional de las riberas de una región es producto de ensambles locales poco diversos pero diferentes entre sí o, en el extremo opuesto, si la diversidad está concentrada a escala local y se repite en toda la región.

Las riberas de cursos de agua que atraviesan pastizales han recibido menos atención que las de áreas boscosas, aun cuando estos ecosistemas herbáceos fueron muy modificados por actividades productivas. Así, los pastizales naturales son transformados en sistemas agrícolas, con el consiguiente desplazamiento, en muchos casos, del ganado hacia los humedales. El pastizal de la región Pampeana está entre los más productivos del mundo y presenta una larga historia de disminución de su diversidad puesto que la vegetación natural, en su mayor parte, ha sido reemplazada por pasturas o por monocultivos de cereales y oleaginosas. Este proceso se ha acentuado en las últimas décadas por la intensificación agrícola. Como consecuencia, algunos pequeños hábitats lineales cercanos a los alambrados y las riberas de los arroyos adquieren importancia como reservorios de la biodiversidad regional. No obstante, la información disponible acerca de la diversidad vegetal de aquéllas es escasa (Faggi et al. 1999; Giorgi 2014; Gantes et al. 2014).

Las principales perturbaciones de las riberas en la Pampa Ondulada son la invasión de acacia negra (Gleditsia triacanthos L.) y la presencia de ganado vacuno. Ambos fenómenos se han acelerado en los últimos años, por un lado a raíz de las características de la acacia que le permiten expandir su área de distribución rápidamente y por el otro, a causa de la intensificación de la agricultura que desplaza la explotación ganadera a zonas bajas (Di Bella et al. 2014) y favorece el uso de los arroyos como abrevaderos. En este trabajo no se consideraron los sitios con acacias, donde las comunidades herbáceas son distintas por estar sometidas a presiones ambientales diferentes a las del pastizal, pero sí se evaluaron los cambios en la vegetación asociados a la presencia de ganado.

Esta investigación tiene como objetivos a) caracterizar la vegetación de las riberas de acuerdo con sus grupos biológicos y b) analizar su diversidad a tres escalas espaciales (margen, tramo y cuenca), analizar la relación de la vegetación con las principales variables topográficas y edáficas de las riberas, y relacionar las características de la vegetación con la presencia de ganado.

\section{Materiales y Métodos}

\section{Área de estudio}

Las riberasestudiadas corresponden a arroyos ubicados en la porción bonaerense de la Pampa Ondulada, subdivisión de los Pastizales del Río de la Plata (Bilenca and Miñarro 2004). De acuerdo con la zonificación realizada por la Subsecretaría de Recursos Hídricos (2010), los arroyos pertenecen a la región hídrica del Noreste de la Provincia de BA, subregiones Cuencas de Arroyos del NE de la Provincia de Buenos Aires y Cuenca de Desagüe al Río de la Plata hasta el río Samborombón. La región se caracteriza por precipitaciones anuales de $\sim 1000 \mathrm{~mm}$ y temperaturas medias entre $14 \mathrm{y}$ $20{ }^{\circ} \mathrm{C}$. El relieve es suavemente ondulado, con un drenaje bien desarrollado hacia el río Paraná y el Río de la Plata, que se manifiesta en numerosos cursos de agua. Analizamos las riberas de arroyos de zonas rurales ubicadas al $\mathrm{N}$ y al S de la región Metropolitana de

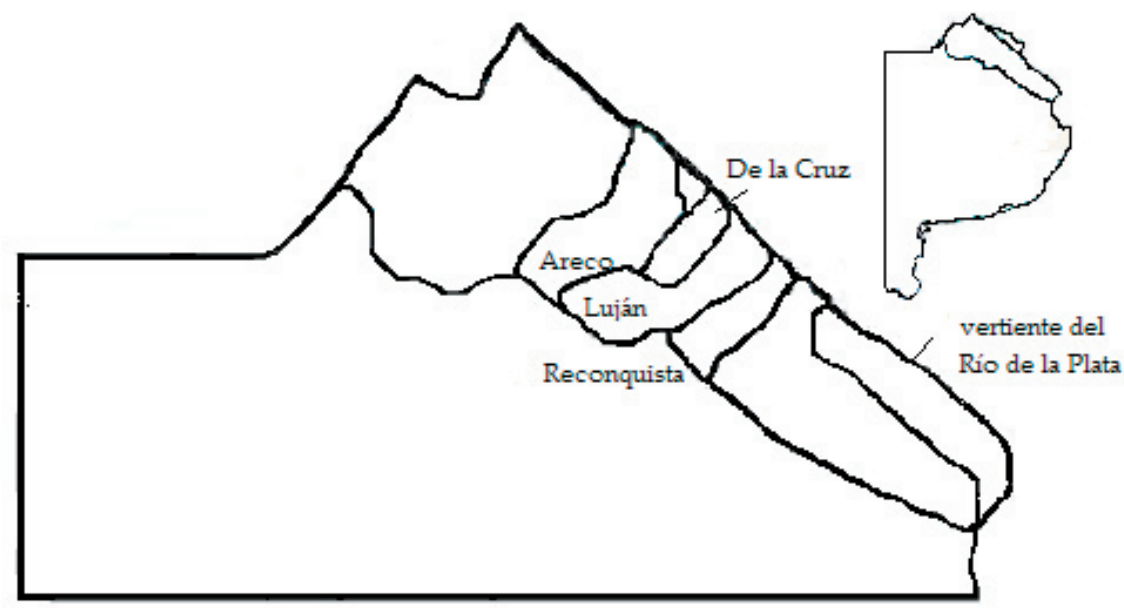

Figura 1. Ubicación de las cuencas donde se realizó el muestreo en la Región Hídrica Noreste de la Provincia de Buenos Aires. Modificado a partir de Giraud et al. 2007.

Figure 1. Localization of the sampled watersheds of the Región Hídrica Noreste de la Provincia de Buenos Aires. 
Buenos Aires. Cuatro de las cuencas (Areco, De la Cruz, Luján y Reconquista) desaguan en el Paraná y la restante (vertiente del Río de la Plata), en el río homónimo (Figura 1). Los arroyos son de órdenes entre 1 a 3 (Strahler) en los lugares de muestreo y presentan crecientes aperiódicas debidas a eventos de precipitación variables en intensidad y en general breves (desde algunas horas hasta uno o dos días).

\section{Obtención y tratamiento de muestras}

Se seleccionaron 14 arroyos pertenecientes a cinco cuencas o vertientes de la Pampa Ondulada: Luján (arroyos Chaña, Haras y Oro), Areco (arroyos Gómez, Vagues y Cañada Honda), De La Cruz (dos arroyos del mismo nombre), Reconquista (Durazno y Nutrias) y Río de la Plata (Espinillo, Juan Blanco y Buñirigo). Se consideraron tres escalas: a) margen, conformada por una franja de ribera de $40 \mathrm{~m}$ de largo por $10 \mathrm{~m}$ de ancho $(\mathrm{n}=27)$, b) tramo, conformado por las dos franjas de $400 \mathrm{~m}^{2}$ separadas por el cauce del arroyo $(n=14)$, a excepción del arroyo Oro, que sólo se muestreó sobre una de las márgenes, y c) cuenca, que comprende dos o tres tramos, cada uno perteneciente a dos o tres arroyos diferentes de una misma cuenca hídrica $(n=5)$. Los arroyos de cada cuenca no están comunicados entre sí, excepto para el arroyo Nutrias, en que se consideraron dos tramos separados por $500 \mathrm{~m}$.

En cada arroyo se muestreó en los meses de noviembre y diciembre, en las dos márgenes de cada tramo de $40 \mathrm{~m}$. Los tramos fueron elegidos por sus condiciones de accesibilidad, y se evitaron aquellos cubiertos por árboles o con uso industrial, y siempre alejados en más de $50 \mathrm{~m}$ de los puentes. En cada margen dentro del tramo se ubicaron seis transectas perpendiculares al curso de agua, sobre las que se midió la cobertura lineal por especie medida en centímetros. El número de transectas fue determinado en un trabajo anterior en base a 14 arroyos de la misma ecorregión (Gantes et al., observación personal). La nomenclatura de los taxa y la asignación a los distintos grupos biológicos responde a Zuloaga and Morrone (1996, 1999a , 1999b); Zuloaga et al. (1994) y a Tur and Lavigne (1992). Los especímenes de dudosa determinación fueron excluidos de los análisis de datos. Ejemplares de cada taxón se hallan depositados en el Laboratorio de Ecología del Departamento de Ciencias Básicas de la Universidad Nacional de Luján.

Para describir la topografía de la ribera se midieron las pendientes transversales al cauce en dos transectas de cada margen. Se utilizó una manguera cristal rellena con agua, con la que se determinaron las diferencias de altura a distancias crecientes de la orilla; por diferencia entre el nivel máximo de referencia y el nivel del agua en cada punto de interés se obtiene la altura con respecto al nivel de la orilla. A partir del cociente entre la altura y la distancia a la orilla se calculó la pendiente para 1, 5 y $10 \mathrm{~m}$ de distancia respecto al cauce. Las muestras de suelo se tomaron también a 0,5 y $10 \mathrm{~m}$, pero en cada una de las seis transectas donde se midió la cobertura vegetal. En cada punto se tomaron dos muestras a $5 \mathrm{~cm}$ de profundidad: en una de ellas se determinó la densidad aparente, con un cilindro de volumen conocido (Chan 2002), y con la muestra restante se determinó el contenido de materia orgánica por el método de Walkey y Black (Jackson 1982), el pH (método potenciométrico, Mettler Toledo MP 220), la conductividad eléctrica por conductimetría (Oakton CON 2700) y el fósforo biodisponible, por el método de Kurtz y Bray (Jackson 1982). Para cada margen se determinó el uso ganadero de la ribera por la presencia de fecas bovinas en el tramo de $400 \mathrm{~m}^{2}$.

\section{Tratamiento de datos}

Para el análisis de la diversidad, en la escala de margen se determinó la riqueza y el índice de Shannon exponencial. Con el objeto de establecer la confiabilidad de los valores de riqueza específica, las curvas obtenidas a partir de las seis transectas en cada margen fueron aleatorizadas utilizando el programa Estimates (8.20) (Colwell 2006) y ajustadas a una función exponencial negativa (Soberón and Llorente 1993):

$$
S_{t}=\frac{a}{b} *\left(1-e^{-b t}\right)
$$

Donde el cociente $a / b$, asíntota de la función exponencial, es el valor de la riqueza estimada.

La diversidad beta para las escalas de margen y tramo se calculó a partir de la expresión de Whittaker (1972):

$$
\text { Diversidad beta }=\frac{S_{c}}{\bar{S}}
$$

donde, para la escala de margen, $S_{c}$ es la riqueza del tramo y $\bar{S}$ es la riqueza promedio de las márgenes; para la escala de tramo, $S_{c}$ es la riqueza de la cuenca y $\overline{\mathrm{S}}$ es la riqueza promedio de los tramos de la cuenca. Para 
comparar los índices de diversidad o la riqueza de especies se utilizó la prueba de KruskalWallis, seguida por comparaciones múltiples y por la prueba U de Mann-Whitney. Se analizó la distancia ecológica entre los ensambles de las cuencas considerando sólo las especies con una abundancia relativa superior a $0.1 \%$, por aplicación del coeficiente de distancia de Bray-Curtis en la escala de margen $(n=27)$, y posterior aplicación de un ordenamiento NMDS y puesta a prueba de la diferencia entre cuencas por ANOSIM (Clark and Warwick 2001).

El análisis de la variación de los factores morfoedáficos se hizo a escala de margen por análisis de componentes principales, y a escala de cuenca se aplicó un análisis discriminante, previa comprobación de la homogeneidad de varianzas de cada variable. Para todos los análisis estadísticos se utilizó el programa Statistica 7.0 (StatSoft, Inc., Tulsa, Oklahoma, USA).

\section{Resultados}

La vegetación de las riberas de los arroyos muestreados es casi exclusivamente herbácea, con $51 \%$ de especies perennes, $27 \%$ de hidrófilas (anfibias y acuáticas), 18\% de anuales y 3\% de árboles. Se identificaron 130 taxa: 120 especies, nueve a nivel de género, una de familia y tres dicotiledóneas que no se pudieron determinar. Ocasionalmente, se encontraron árboles (Celtis ehrenbergiana (Klotzsch) Liebm. y Salix humboldtiana Willd., nativos, y Gleditsia triacanthos L. y Morus alba L., introducidos). Trece taxa fueron frecuentes (presentes en más de 50\% de las márgenes) y abundantes (más de 50\% de cobertura promedio), y entre ellos, Cynodon dactylon(L.) Pers. y Eleocharis spp. presentaron frecuencias cercanas al $100 \%$ y los más altos valores de cobertura: 29 y $8 \%$, respectivamente. Un $76 \%$ de las especies identificadas son nativas, endémicas o cosmopolitas, y el $24 \%$ son introducidas, naturalizadas o adventicias. El 97\% de las especies anfibias y acuáticas, el $63 \%$ de las perennes y el 36\% de las anuales son nativas.

La riqueza estimada del conjunto de 27 márgenes (diversidad gama) fue de 130 taxa (DE=3.01). La diversidad alfa promedio observada por margen de $400 \mathrm{~m}^{2}$ fue 24.4 especies ( $\mathrm{DE}=8.45)$, siendo en el $74 \%$ de los sitios superior a $95 \%$ de la riqueza estimada por la función exponencial negativa. La vertiente del Río de La Plata fue la más diversa (Índice de Shannon exponencial), y se distinguió significativamente $(P<0.05)$ de la cuenca del río Areco (Figura 2). La diversidad beta entre la escala de margen y la de tramo fue, en promedio, $1.37(\mathrm{n}=13 ; \mathrm{DE}=0.07)$. Esto significa que el número de especies del tramo fue 1.37 veces el de una sola margen, mientras que la beta entre tramos y cuencas fue 1.89 $(\mathrm{n}=5 ; \mathrm{DE}=0.24)$.

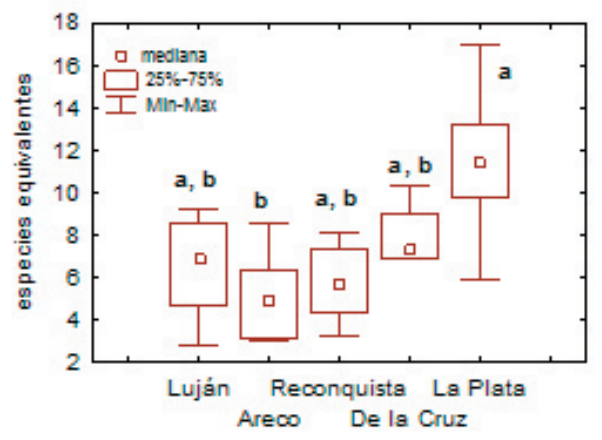

Figura 2. Diversidad (índice de Shannon exponencial) de las cinco cuencas. Prueba de Kruskal-Wallis seguida de comparaciones múltiples, letras distintas indican diferencias significativas $(P<0.05)$.

Figure 2. Diversity (Shannon exponential index) of the five watersheds. Kruskal-Wallis test followed by multiple comparisons; different letters indicate significant differences $(P<0.05)$.

Entre los grupos biológicos, a escala de margen, las perennes tienen la mayor diversidad alfa, seguidas por las acuáticas y las anuales $(P<0.01)$; a escala de tramo, sólo se distinguen las especies perennes $(P<0.01)$ (Figura 3). Por su diversidad beta sólo se distinguieron acuáticas de anuales a escala de tramo $(P<0.05)$ (Figura 4$)$.

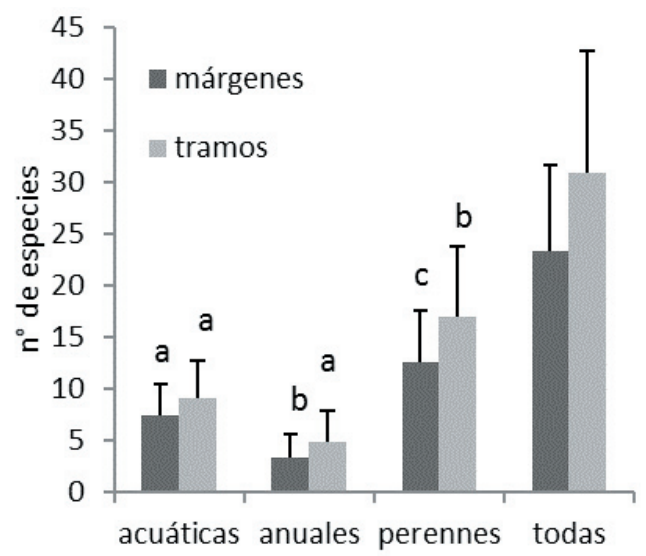

Figura 3. Riqueza de especies en márgenes y tramos. Letras distintas indican diferencias significativas $(P<0.05)$ dentro de cada escala (Kruskal-Wallis y comparaciones múltiples).

Figure 3. Species richness of streambanks and reaches. Different letters indicate significant differences $(P<0.05)$ (Kruskal-Wallis and multiple comparisons). 
Tabla 1. Valores medios y coeficientes de variación de las variables medidas en la escala de margen, y promedios para cada cuenca (DLC: De La Cruz; LUJ: Luján; ARE: Areco; RLP: Río de la Plata; REC: Reconquista).

Table 1. Mean values and coefficient of variation of the variables measured at streambank scale, and averages of each watershed (DLC: De La Cruz; LUJ: Luján; ARE: Areco; RLP: Río de la Plata; REC: Reconquista).

\begin{tabular}{lccccccc}
\hline Variable & Promedio & $\mathrm{CV}$ & DLC & LUJ & ARE & RLP & REC \\
\hline Densidad aparente $\left(\mathrm{g} / \mathrm{cm}^{3}\right)$ & 1.4 & 0.09 & 0.80 & 0.71 & 0.80 & 3.64 & 0.74 \\
$\mathrm{Ph}$ & 7.91 & 0.03 & 7.57 & 8.30 & 7.60 & 7.81 & 8.24 \\
Conductividad $(\mu \mathrm{S} / \mathrm{cm})$ & 517.05 & 0.24 & 742.36 & 413.99 & 301.66 & 613.42 & 571.74 \\
Fósforo biodisponible $(\mathrm{ppm})$ & 18.67 & 0.39 & 14.30 & 16.57 & 11.44 & 6.60 & 42.65 \\
Materia orgánica $(\%)$ & 4.11 & 0.25 & 5.06 & 3.97 & 3.68 & 3.78 & 4.38 \\
Pendiente entre 0 y 1 m & 0.57 & 0.32 & 0.63 & 0.39 & 0.55 & 0.58 & 0.70 \\
Pendiente entre 0 y 5 m & 0.19 & 0.15 & 0.23 & 0.20 & 0.20 & 0.21 & 0.25 \\
Pendiente entre 0 y 10 m & 0.14 & 0.2 & 0.16 & 0.13 & 0.12 & 0.11 & 0.14 \\
Pendiente máxima & 0.64 & 0.21 & 0.66 & 0.44 & 0.65 & 0.67 & 0.75 \\
\hline
\end{tabular}

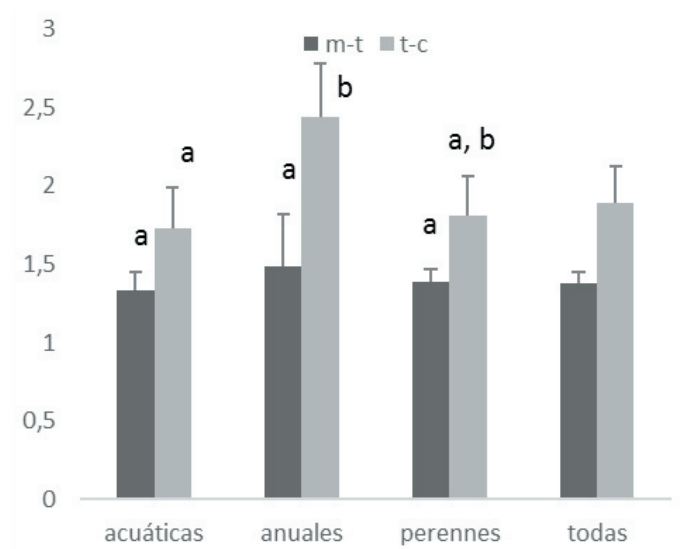

Figura 4. Diversidad beta entre márgenes y tramos $(m-t)$ y entre tramos y cuencas $(\mathrm{t}-\mathrm{c})$. Letras distintas indican diferencias significativas $(P<0.05)$ dentro de cada escala (Kruskal-Wallis y comparaciones múltiples).

Figure 4. Beta diversity between streambanks and reaches $(\mathrm{m}-\mathrm{t})$, and between reaches and watersheds $(\mathrm{t}-\mathrm{c})$. Different letters indicate significant differences $(P<0.05)$ among each scale (Kruskal-Wallis and multiple comparisons).

El análisis de los ensambles de vegetación de las cuencas mediante un ordenamiento NMDS y posterior ANOSIM no permitió diferenciarlos (no se muestra). La Tabla 1 muestra los valores medios de las variables edáficas y las pendientes de las 27 márgenes relevadas. Se observa que los arroyos son encajonados, con una pendiente abrupta en el primer metro adyacente a la costa (0.57), y luego suavizada hasta los $10 \mathrm{~m}$ de distancia al agua.

En relación al margen, en el análisis de componentes principales, la variación sobre los dos primeros ejes alcanzó el 54\% del total, donde se distingue un eje morfométrico (33\%) y un eje asociado a las variables edáficas (21\%) (Figura 5). Para el eje 1, la contribución de las pendientes medidas a 5 y $10 \mathrm{~m}$ y la pendiente máxima fue similar y más alta

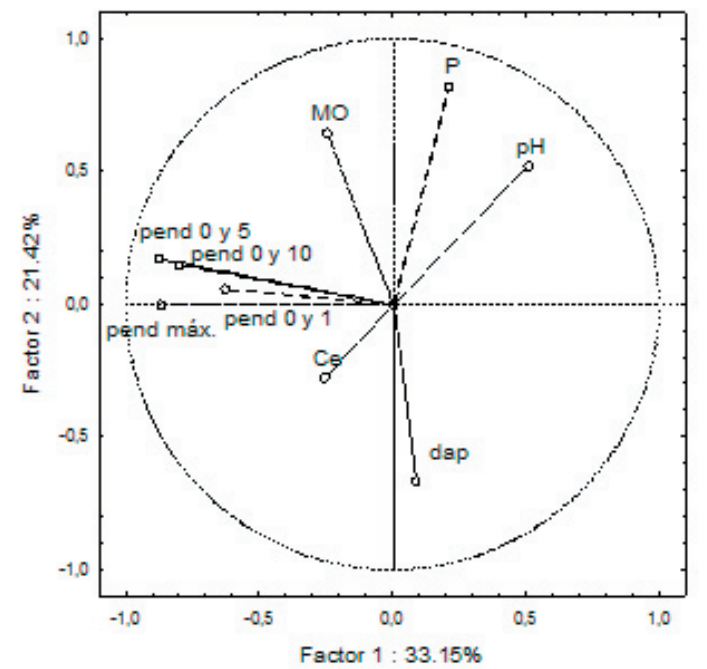

Figura 5. Análisis de componentes principales de las variables morfoedáficas. Representación en el espacio de los ejes factores 1 y 2 de las siguientes variables: dap (densidad aparente), Ce (conductividad eléctrica), $\mathrm{P}$ (fósforo biodisponible), MO (materia orgánica [\%]), pendiente entre $0 \mathrm{y} 1 \mathrm{~m}$, pendiente entre 0 y $5 \mathrm{~m}$, pendiente entre 0 y $10 \mathrm{~m}$ y pendiente máxima.

Figure 5. Principal components analysis of morphoedaphic variables. Representation in the space of factors 1 and 2 of variables: dap (bulk density), Ce (conductivity), $\mathrm{P}$ (bioavailable phosphorus), MO (organic matter [\%]), slopes between 0 and 1,5,10 $\mathrm{m}$ and maximum slope, respectively: Pend 0 and 1 , Pend 0 and 5, Pend 0 and 10, and Pend Max.

que la medida en las proximidades del agua. Entre las características edáficas, el fósforo y la densidad aparente aportaron la mayor variabilidad sobre el eje 2 , pero en sentidos opuestos.

En el análisis discriminante, la primera función distinguió las cuencas por un $99 \%$ $\left(\mathrm{Chi}^{2}=132.8 ; \quad P<0.01\right)$; la vertiente Río de la Plata quedó separada de las otras cuatro (Figura 6). La densidad aparente es la variable que más contribuye a la separación, 


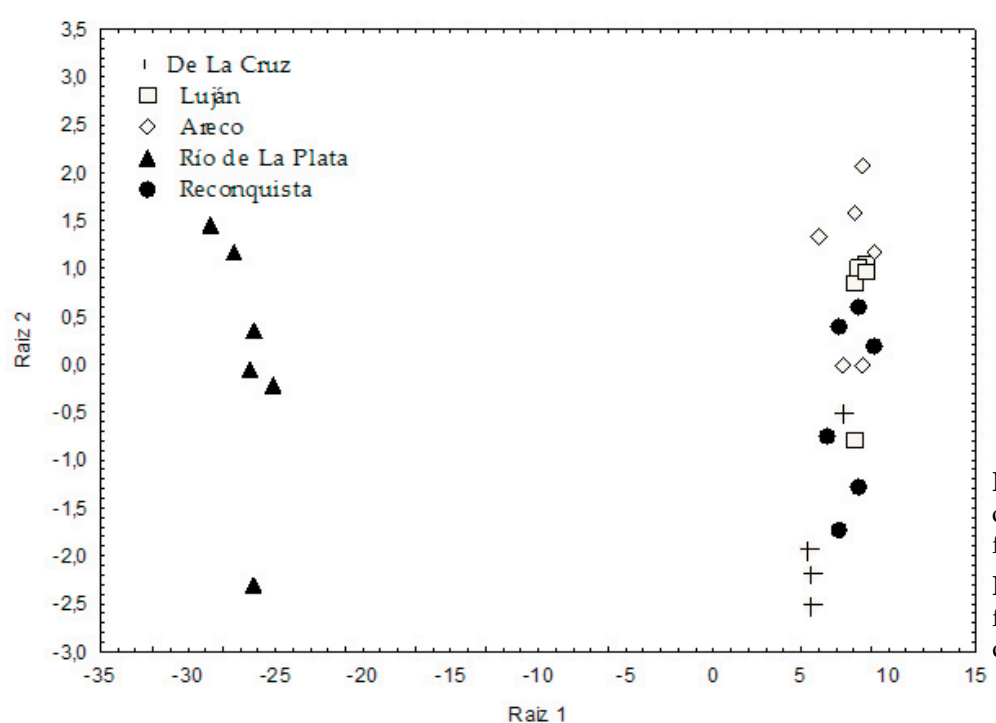

aunque la materia orgánica también resultó significativa (pero con un aporte pequeño para la segregación [Tabla 2]). En este análisis se excluyó al fósforo por no cumplir el supuesto de homogeneidad de varianzas. La diversidad se correlacionó significativa e inversamente con los escores del segundo eje principal del análisis de componentes principales ( $\mathrm{r}=-0.51)$, y entre las características ambientales, con la densidad aparente ( $r=0.63$ ) (Tabla 3).

Tabla 2. Análisis discriminante. Contribución de las variables a la separación de las cuencas.

Table 2. Discriminant analysis. Variables' contribution to the segregation of watersheds.

\begin{tabular}{lccc}
\hline \multicolumn{1}{c}{ Variable } & $\begin{array}{c}\lambda \text { de Wilks } \\
\text { parcial }\end{array}$ & F & $P$ \\
\hline Densidad aparente & 0.005 & 728.25 & 0.00 \\
$\mathrm{pH}$ & 0.739 & 1.32 & 0.30 \\
Conductividad & 0.582 & 2.68 & 0.07 \\
Materia orgánica & 0.461 & 4.37 & 0.01 \\
Pend, entre 0 y 1m & 0.937 & 0.24 & 0.90 \\
Pend, entre 0 y 5 m & 0.825 & 0.79 & 0.54 \\
Pend, entre 0 y 10m & 0.785 & 1.02 & 0.42 \\
Pend, máxima & 0.927 & 0.29 & 0.87 \\
\hline
\end{tabular}

Tabla 3. Correlaciones entre las variables ambientales y el índice de Shannon exponencial. ${ }^{*}=P<0.05$.

Table 3. Correlations between environmental variables and Shannon exponential index. ${ }^{*}=P<0.05$.

\begin{tabular}{lc}
\hline \multicolumn{1}{c}{ Variable } & r de Pearson \\
\hline Densidad aparente & $0.63^{*}$ \\
Ph & -0.14 \\
Conductividad & 0.18 \\
Fósforo biodisponible & -0.36 \\
Materia orgánica & -0.08 \\
Pendiente entre 0 y 1 m & -0.16 \\
Pendiente entre 0 y 5 m & -0.16 \\
Pendiente entre 0 y $10 \mathrm{~m}$ & -0.06 \\
Pendiente máxima & -0.13 \\
\hline
\end{tabular}

Figura 6. Datos multivariados de las cinco cuencas en el espacio de las funciones discriminantes 1 y 2 .

Figure 6. Multivariate data of the five watersheds in the space of discriminant functions 1 and 2 .

\section{DisCUSIÓN}

En general, las riberas son consideradas zonas de alta riqueza específica y de refugio para especies nativas (Naiman et al.1993; Boutin et al. 2003), aunque también funcionan como corredores para exóticas (Planty-Tabacchi et al. 1996; Sabo et al. 2005; Richardson et al. 2007; Bowers and Boutin 2008; Magee et al. 2008). Encontramos un promedio de 24 especies en áreas de $400 \mathrm{~m}^{2}$, con una representación de especies exóticas de $24 \%$. Este porcentaje es muy similar a otras proporciones señaladas para habitats no ribereños de Buenos Aires: $21 \%$ para los pastizales de la Pampa Deprimida, 23\% para los alrededores de Buenos Aires y $25 \%$ para toda la provincia (Chaneton et al. 2002), de modo que las riberas no serían ambientes particularmente refractarios a la presencia de especies exóticas. Sin embargo, su valor de conservación se ha relacionado no sólo con el número de especies, sino además con el hecho de que albergan un conjunto de taxa exclusivos que incrementan la biodiversidad regional (gamma) a través de un alto valor de diversidad beta (Sabo et al. 2005). Si bien no contamos con datos acerca de los sitios no ribereños, la proporción de especies hidrófilas fue del $27 \%$, y podemos considerar esta cifra como un indicio del aporte mínimo aproximado de las zonas ribereñas a la biodiversidad regional. Pero, además, las especies hidrófilas son casi exclusivamente nativas $(97 \%)$, lo que aumenta la importancia de su conservación. Por otro lado, este grupo biológico tiene relevancia funcional, pues son más eficientes en la retención de sedimentos (Micheli and Kirchner 2002; De Keiser et al. 2003). Santos (2010) plantea que como consecuencia de los disturbios antrópicos, 
la vegetación de zonas altas avanza sobre las especies características de las riberas. En nuestro caso, la alta proporción de especies mesófilas puede relacionarse con el perfil encajonado de los arroyos de cabecera, donde hay una baja influencia (en frecuencia y duración) de las crecientes sobre las márgenes.

Encontramos una diversidad beta promedio de 1.37 entre márgenes y tramos, y de 1.8 entre tramos y cuencas, valores que indican un incremento importante de la riqueza de especies con la escala. La diversidad beta es similar para los distintos grupos biológicos entre márgenes y tramos, pero entre tramos y cuencas, las hidrófilas tienen una beta menor que las anuales. Esto indicaría que las riberas de los tramos de una misma cuenca resultan más homogéneas para las hidrófilas que para las anuales.

El grupo de variables morfométricas (pendientes) es el que más contribuye a separar los sitios en la escala de margen (primer eje del análisis de componentes principales). De las distintas pendientes calculadas se observa que la correspondiente al primer metro adyacente al cauce contribuye en menor proporción que el resto a explicar la variación entre márgenes. De este resultado se desprende que para una caracterización adecuada de los tramos se podrían utilizar indistintamente la pendiente medida entre 0 y $5 \mathrm{~m}$ ó entre 0 y $10 \mathrm{~m}$, pero no la medida entre 0 y $1 \mathrm{~m}$.

Sin embargo, la diversidad alfa está más relacionada con las variables edáficas (segundo eje principal) que con las pendientes y, además, la correlación más alta se observó con la densidad aparente $(r=0.64)$. La cuenca del Río de la Plata es la más diversa a pesar de que el análisis de los ensambles de las cuencas mediante un ordenammiento NMDS y posterior ANOSIM no permitió diferenciarlos. Esta cuenca reúne 88 especies, 27 de las cuales son exclusivas de esta cuenca, y se separa del resto en el análisis discriminante. En los suelos de esta vertiente, los horizontes superficiales presentan una textura franco-limosa y un horizonte argílico, lo cual justifica los altos valores en la densidad aparente, a diferencia de los suelos de las restantes cuencas, que son profundos y sin presencia del horizonte argílico (Subsecretaría de Recursos Hídricos 2010). Esta relación positiva entre la densidad aparente y la diversidad no es esperable pues valores superiores a $1.65 \mathrm{~g} / \mathrm{cm}^{2}$ en suelos franco arcillo-limosos, como los observados en la vertiente del Río de la Plata, dificultan el desarrollo de las raíces (USDA 2015). Esto supone un menor número de especies capaces de establecerse en sitios con esa condición de estrés ambiental.

La comparación entre tramos respecto del uso ganadero se hizo mediante la cobertura relativa de exóticas, que se ha señalado como más efectiva que las medidas de diversidad para indicar el disturbio antropogénico (Bowers and Bowtin 2008), y también mediante el índice de Shannon exponencial. En ninguno de los dos casos hubo diferencias entre los tramos. La riqueza de especies fue mayor en los sitios con ganado. Este resultado, que también encontramos al comparar dos tramos de uno de los arroyos muestreados en este trabajo (Gantes et al. 2014), ha sido observado por otros autores, quienes refieren mayor riqueza de especies en sitios bajo uso ganadero que en pastizales naturales, o bien muestran cambios en su composición florística (e.g., Jansen and Robertson 2001; Jackson and Allen-Díaz 2006; Burnside et al. 2007; Di Bella 2014). La diferencia entre las estimaciones de la diversidad (Shannon exponencial y riqueza) sugieren una mayor importancia relativa de las especies poco abundantes en los sitios con ganado. De todos modos, no es el uso actual sino la historia de uso en el lugar el factor que podría dar cuenta de la composición de los ensambles vegetales, y no se cuenta con esa información.

Los ecosistemas de ribera se caracterizan por los disturbios frecuentes ligados a la dinámica fluvial. No obstante, en las riberas de los arroyos que muestreamos encontramos sólo $27 \%$ de especies hidrófilas (anfibias y acuáticas), lo que se relaciona con un régimen de crecientes poco frecuentes, pero principalmente de pequeña duración. Pese a contar con un número minoritario de especies, la importancia de este grupo radica en que son propias de estos ambientes, casi todas nativas y tienen características funcionales relevantes para la estabilidad morfológica de las riberas. Por otra parte, el aumento de la diversidad en valores cercanos al doble cuando la escala pasa de tramos a cuencas indica que la componente reemplazo de especies de la diversidad beta contribuye de manera importante a la biodiversidad regional. Esto debe tenerse en consideración al momento de diseñar investigaciones y también en las estrategias de manejo que no deberían focalizarse en la preservación puntual de algunos tramos sino en una interpretación del funcionamiento de las riberas a escala de cuenca. 
Agradecimientos. A L. Giménez, A. Mónaco, A. Nistal, C. Lallana y Ceci Hegoburu por su trabajo de campo, laboratorio y gabinete. Por su colaboración en la determinación de algunos ejemplares agradecemos a Greppi, A. Escobar, M. Rodríguez Morcelle y agradecemos a R. Dubois por su colaboración en la determinación de las pendientes.

\section{REFERENCIAS}

Bilenca, D., and F. Miñarro. 2004. Identificación de áreas valiosas de pastizal (AVPs) en las Pampas y Campos de Argentina, Uruguay y sur de Brasil. Fundación Vida Silvestre. Argentina. Pp. 323.

Boutin, C., J. Benoit, and C. Bélanger. 2003. Importance of riparian habitats to flora conservation in farming landscapes of southern Québec, Canada. Agriculture, Ecosystems and Environment 94:73-87.

Bowers, K., and C. Boutin. 2008. Evaluating the relationship between floristic quality and measures of plant biodiversity along stream bank habitats. Ecol Indic 8(5):466-475.

Burnside, N., C. B. Joyce, E. Puurmann, and D. M. Scott. 2007. Use of Vegetation Classification and Plant Indicators to Assess Grazing Abandonment in Estonian Coastal Wetlands. Journal of Vegetation Science 18(5):645-654.

Chan, K. Y. 2002. Bulk density. Encyclopedia of soil science. Lal, R., ed. $2^{\circ}$ ed. Marcel Dekker, New York, NY, USA.

Chaneton, E. J., S. B. Perelman, M. Omacini, and R. J. C. León. 2002. Grazing, environmental heterogeneity, and alien plant invasions in temperate Pampa grasslands. Biol Invasions 4:7-24.

Clarke, K. R., and R. M. Warwick. 2001. Changes in marine communities: an approach to statistical analysis and interpretation. PRIMERE: Plymouth.

Colwell, R. K. 2006. EstimateS: statistical estimation of species richness and shared species from samples Version 8.2. Persistent URL: purl.oclc.org/estimates.

De Keyser, E. S., D. R. Kirby, and M.J. Ell. 2003. An index of plant community integrity: development of the methodology for assessing prairie wetland plant communities. Ecol Indic 3:119-133.

Di Bella, C. E., E. Jacobo, R. A. Golluscio, and A. M. Rodríguez. 2014. Effect of cattle grazing on soil salinity and vegetation composition along an elevation gradient in a temperate coastal salt marsh of Samborombón Bay (Argentina). Wetl Ecol Manag 22:1-13.

Faggi, A. M., M. O. Arriaga, and S.S. Aliscioni. 1999. Composición florística del río Reconquista y sus alteraciones antrópicas. Revista del Museo Argentino de Ciencias Naturales 1(1):1-6.

Gantes, P., L. Falco, and A. Sánchez Caro. 2014.Efecto del ganado sobre la estructura de la vegetación en las riberas de un arroyo pampeano. Biología Acuática 30:123-131.

Giling, D. P., M. R. Grace, J. R. Thomson, R. Mac Nally, and R. M. Thompson. 2014. Effect of native vegetation loss on stream ecosystem processes: dissolved organic matter composition and export in agricultural landscapes. Ecosystems 17:82-95.

Giorgi, A., J. J. Rosso, and E. Zunino. 2014. Efectos de la exclusión de ganado sobre la calidad ambiental de un arroyo pampeano. Biología Acuática 30:133-140.

Giraud, M. A., R. L. Aguglino, C. Lupano, E. Bozzarello, J. M. Cornejo, and C. Rey. 2007. Regiones hídricas superficiales de la provincia de Buenos Aires - Actualización cartográfica digital. XII Congreso de la Asociación Española de Teledetección.

Gurnell, A. 2014. Plants as river system engineers. Earth Surf Proc Land 39(1):4-25.

Hood, W. G, and R. J. Naiman. 2000. Vulnerability of riparian zones to invasion by exotic vascular plants. Plant Ecol 148(1):105-114.

Jackson, L. M. 1982. Análisis químico de suelos. Editorial Omega. Barcelona, España. Pp. 682.

Jackson, R. D., and B. Allen-Diaz. 2006. Spring-fed wetland and riparian plant communities respond differently to altered grazing intensity. J Appl Ecol 43:485-498.

Jansen, A., and A. I. Robertson. 2001. Relationships between livestock management and the ecological condition of riparian habitats along an Australian floodplain river. J Appl Ecol 38:63-75.

Magee, T., P. Ringold, and M. Bollman. 2008. Alien species importance in native vegetation along wadeable streams, John Day River basin, Oregon, USA. Plant Ecol 195:287-307.

Micheli, E. R., and J. W. Kirchner. 2002. Effects of wet meadow riparian vegetation on streambank erosion. 1. Remote sensing measurements of streambank migration and erodibility. Earth Surf Proc Land 27:627-639.

Naiman, R. J., H. Decamps, and M. Pollock. 1993. The role of riparian corridors in maintaining regional biodiversity. Ecol Appl 3(2):209-212.

Naiman, R. J., R. E. Bilby, and P. A. Bisson. 2000. Riparian ecology and management in the Pacific coastal rain forest BioScience 50(11):996-1011.

Nilsson C., and M. Svedmark. 2002. Basic principles and ecological consequences of changing water regimes: riparian plant communities. Environ Manage 30:468-480.

Osborne L. L., and D. A. Kovacic. 1993. Riparian vegetated buffer strips in water-quality restoration and stream management. Freshwater Biol 29:243-258.

Planty Tabacchi, A. M., E. Tabacchi, R. J. Naiman, C. Deferrari, and H. Decamps. 1996. Invasibility of species-rich communities in riparian zones. Conserv Biol 10(2):598-607. 
LeRoy Poff, N., J. D. Allan, M. B. Bain, J. R. Karr, K. L. Prestegaard, et al. 1997. The Natural Flow Regime A paradigm for river conservation and restoration Bioscience 47(11):769-784.

Richardson, D. M., P. M. Holmes, K. J. Esler, S. M. Galatowitsch, J. C. Stromberg, et al. 2007. Riparian vegetation: degradation, alien plant invasions, and restoration prospects. Divers Distr 13:126-139.

Sabo, J., R. Sponseller, M. Dixon, K. Gade, T. Harms, et al. 2005. Riparian zones increase regional species richness by harboring different, not more, species. Ecology 86(1):56-62.

Santos, M. J. 2010. Encroachment of upland Mediterranean plant species in riparian ecosystems of southern Portugal. Biodivers Conserv 19:2667-2684.

Soberon, M., and J. Llorente. 1993. The Use of Species Accumulation Functions for the Prediction of Species Richness. Conserv Biol 7(3):480-488.

Subsecretaría de Recursos Hídricos. 2010. Atlas de Cuencas y Regiones Hídricas Superficiales de la República Argentina. Versión 2010.

Tur, M. N., and A. Lavigne. 1992. Plantas acuáticas de la provincia de Buenos Aires. Situación Ambiental de la provincia de Buenos Aires. A. Recursos y rasgos naturales en la evaluación ambiental. Comisión de Investigaciones Científicas y Técnicas de la Provincia de Buenos Aires 18, La Plata, Argentina.

USDA. 2015. www.nrcs.usda.gov/wps/portal/nrcs/detail/soils/ref/?cid=nrcs142p2_054223. Última fecha de ingreso abril 2016.

Ward, J. V., K. Tockner, D. B. Arscott, and C. Claret. 2002. Riverine landscape diversity. Freshwater Biol 47:517-539.

Whittaker, R. H. 1972. Evolution and Measurement of Species Diversity. Taxon 21(2/3):213-251.

Young-Mathews, A., S. W. Culman, S. Sánchez-Moreno, A. T. O'Geen, H. Ferris, et al. 2010. Plant-soil biodiversity relationships and nutrient retention in agricultural riparian zones of the Sacramento Valley, California. Agroforest Syst 80:41-60.

Zuloaga, F. O., and O. Morrone. 1996. Catálogo de las Plantas Vasculares de la República Argentina. I. Pteridophyta, Gymnospermae y Angiospermae (Monocotyledoneae). Monographs in Systematic Botany from the Missouri Botanical Garden 60:1-323.

Zuloaga, F. O., and O. Morrone. 1999a. Catálogo de las Plantas Vasculares de la República Argentina. II. AcanthaceaeEuphorbiaceae (Dicotyledoneae). Monographs in Systematic Botany from the Missouri Botanical Garden 74:1-621.

Zuloaga, F. O., and O. Morrone. 1999b. Catálogo de las Plantas Vasculares de la República Argentina. II. Fabaceae -Zygophyllaceae (Dicotyledoneae). Monographs in Systematic Botany from the Missouri Botanical Garden 74:11269.

Zuloaga, F. O., E. G. Nicora, Z. Rúgolo de Agrasar, O. Morrone, J. Pensiero, et al. 1994. Catálogo de la Familia Poaceae en la República Argentina. Monographs in Systematic Botany from the Missouri Botanical Garden 47:1-178. 